\title{
Diabetes gestacional assistida: perfil e conhecimento das gestantes
}

\author{
Rosiana Carvalho Costa ${ }^{1}$, Márcia Oliveira Coelho Campos², Lidia Audrey Rocha Valadas Marques³, \\ Edilson Martins Rodrigues Neto ${ }^{4}$, Maria Celsa Franco ${ }^{5}$, Érika Sabóia Guerra Diógenes ${ }^{6}$
}

\begin{abstract}
RESUMO
A gravidez é um estado que pode provocar uma intolerância aos carboidratos e à Diabetes Mellitus (DM). Quando essa doença é diagnosticada durante a gravidez é denominada Diabetes Mellitus Gestacional (DMG). A presença de DMG implica alto risco para mãe e para o recémnascido. $\mathrm{O}$ estudo tem como objetivos: descrever o perfil socioeconômico, epidemiológico e clínico das gestantes portadoras de DMG; Compreender o conhecimento das gestantes portadoras de DMG, atendidas em uma instituição de referência em saúde da mulher no Estado do Ceará, sobre sua patologia. Trata-se de um estudo de natureza qualitativa e quantitativa, com predominância qualitativa, realizado em um hospital público de Fortaleza/Ce, no período de agosto a outubro de 2011, através da aplicação de um questionário. Os dados quantitativos foram analisados através do programa estatístico SPSS e os dados qualitativos através do Método Fenomenológico de Amedeo Giorgi (1985). Os resultados apontam que o diagnóstico de $6(35,4 \%)$ das participantes DMG ocorreu antes da 20a semana de gestação; que das gestantes pesquisadas, $11(64,7 \%)$ relataram que tinham parentes diabéticos, indício preocupante, tendo em vista que o histórico familiar é um fator de risco para desencadear DMG. Observou-se também que 12 (70,06\%) tiveram o pré-natal com quantidade de consulta preconizada pelo Ministério da Saúde, mesmo assim a maioria informou que não foi alertada quanto a DMG. Conclui-se que as gestantes entrevistadas não possuem muita informação sobre sua patologia e possíveis complicações.
\end{abstract}

Descritores: Saúde da mulher; Diabetes Gestacional, Perfil de Saúde.

\section{Diabetes gestational assisted: profile and knowledge of pregnant women}

\begin{abstract}
Pregnancy is a condition that can cause intolerance to carbohydrates when diagnosed during pregnancy is called Gestational Diabetes Mellitus. The presence of Gestational Diabetes Mellitus (GDM) implies high risk for mother and newborn. The study aims to: describe the socioeconomic, clinical and epidemiological profile of pregnant women with gestational diabetes; Understand the knowledge of pregnant women with gestational diabetes treated at a referral institution in women's health in the state of Ceará, about his condition. This is a study of qualitative and quantitative, qualitative predominance conducted in a public hospital in Fortaleza / Ce in the period August to October 2011, through a questionnaire. Quantitative data were analyzed using SPSS and qualitative data through the phenomenological method Amedeo Giorgi (1985). The results indicate that the diagnosis of $6(35.4 \%)$ of participants DMG occurred before the 20th week of gestation; that the women surveyed, $11(64.7 \%)$ reported that they had diabetic relatives, worrying evidence, given that family history is a risk factor for triggering DMG. It was also observed that $12(70.06 \%)$ had prenatal consultation with the amount recommended by the Ministry of Health, yet the majority reported that it was not warned about DMG. We conclude that pregnant women interviewed did not have much information about their condition and possible complications.
\end{abstract}

Descriptors: Women's Health; Diabetes Gestational, Health Profile.

${ }^{1}$ Especialista em Saúde Pública pela Universidade Estadual do Ceará (UECE), Fortaleza, CE, Brasil.

${ }^{2}$ Mestre em Saúde Pública pela Universidade Federal do Ceará (UFC), Fortaleza, CE, Brasil.

${ }^{3}$ Especialista em Farmacologia Clínica pelo Instituto Ateneu , Fortaleza, CE, Brasil.

${ }^{4}$ Mestre em Farmacologia pela Universidade Federal do Ceará (UFC), Fortaleza, CE, Brasil.

${ }^{5}$ Mestre em Enfermagem pela Universidade Estadual do Ceará (UECE), Fortaleza, CE, Brasil.

${ }^{6}$ Farmacêutica pela Universidade Federal do Ceará (UFC), Fortaleza, CE, Brasil. 


\section{Introdução}

É crescente a importância do Diabetes Mellitus (DM) como problema de saúde pública, quer pela magnitude quer pelas complicações, isto em quase todos os países. Essa importância é devido ao aumento de sua prevalência, morbidade e mortalidade ${ }^{1}$. O último estudo da Organização Mundial de Saúde (OMS) estimou que até 2030 o número de pessoas com diabetes será aproximadamente 366 milhões e que ainda neste século, o Brasil terá aproximadamente 11 milhões de indivíduos com essa patologia².

A classificação atual do DM proposta pela Associação Americana de Diabetes (ADA) e adotada pela OMS e Sociedade Brasileira de Diabetes (SBD), é baseada em sua etiologia e não no tipo de tratamento. Inclui quatro classes clínicas: Tipo 1(DM1), Tipo 2(DM2), outros tipos específicos de DM e diabetes mellitus gestacional (DMG), sendo essa definida como qualquer intolerância a glicose, de magnitude variável, diagnosticada ou reconhecida pela primeira vez durante a gravidez².

A gravidez é um estado que pode provocar hiperinsulinemia, sendo caracterizada pelo aumento progressivo da necessidade e da resistência à insulina. Nas mulheres com deficiência parcial ou completa na função das ilhotas de Langerhans, ocorre a intolerância aos carboidratos de qualquer gravidade, quando diagnosticada durante a gravidez é denominada Diabetes Mellitus Gestacional (DMG)3.

A prevalência global média de DMG é de $10 \%$, podendo variar de $1 \%$ a $14 \%$. Essa grande variabilidade vai de acordo com as características da população estudada, como a prevalência de diabetes tipo 2, a composição étnica e com emprego de diferentes diagnósticos adotados. Essa mesma patologia é responsável por $90 \%$ de todas as gestações acometidas pela intolerância a glicose de todos os casos de gestação diabética. A presença de DMG implica alto risco para mãe e para o recém-nascido. A morbidade perinatal é aumentada quando comparada a da população geral de grávidas ${ }^{4}$.

Existe uma associação entre a hiperglicemia materna e a morbidade fetal ${ }^{5}$. 0 recém-nascido de uma mulher com diabetes corre o risco de anoxia, prematuridade, infecção, desconforto respiratório, hipoglicemia grave, hipocalemia, hiperribilirrubinemia, polidrâmnio, macrossomia, policitemia e óbito fetal ${ }^{6}$. Prevalecendo com maior risco, macrossomia e hipoglicemia².

O tratamento do DMG envolve o emprego de dieta adequada, exercício físico e insulina, acompanhada por uma equipe multidisciplinar. Deve-se considerar a idade da gestante, seu estado geral de saúde, sua história médica; na tolerância a certos medicamentos, procedimentos e terapias; na sua expectativa para a trajetória da doença e em mudança no estilo de vida, tais como: uma dieta especial, exercício físico e monitorização da glicose no sangue diariamente e estabelecimento de um peso adequado. A insulina de uma maneira geral só é introduzida quando a dieta e os exercícios não levam a um controle metabólico ${ }^{5}$.

Diante do exposto questiona-se se existe alguma relação do desenvolvimento de diabetes gestacional com o perfil das gestantes portadoras de diabetes gestacional. E, qual a percepção das gestantes portadoras de diabetes gestacional sobre sua doença?

O estudo contribuirá para com os profissionais de saúde no intuito de fornecer subsídios para o desenvolvimento de estratégias de prevenção do diabetes gestacional, a partir da identificação de fatores relacionados ao perfil das gestantes e como as gestantes estão percebendo a doença. Com isso, contribuir para com o SUS na promoção da saúde do binômio mãe e filho.

Para isso objetivou-se nesse trabalho descrever o perfil socioeconômico, epidemiológico e clínico das gestantes portadoras de DMG; compreender o conhecimento das gestantes portadoras dessa doença, atendidas em uma instituição de referência em saúde da mulher no Estado do Ceará, sobre sua patologia.

\section{Metodologia}

Trata-se de um estudo exploratório, de natureza qualitativa e quantitativa, com predominância qualitativa. 0 estudo associou os métodos de pesquisa quantitativos e qualitativos porque eles não são antagônicos, e sim complementares fazendo enriquecer os resultados.

O método de pesquisa qualiquantitativa pressupõem uma análise dialética que permita enxergar que ambas podem ser trabalhadas em conjunto, trazendo elementos que se complementam, corroborando para uma interpretação mais abrangente da realidade posta.

A unidade de pesquisa ocorreu em um hospital geral da rede estadual de saúde do SUS, situado em Fortaleza-CE. O hospital tem como missão prestar assistência ao usuário do SUS, com excelência nos atendimentos Materno-infantil, Clínico, Cirúrgico, Ensino e Pesquisa. 
A população alvo foi composta por 17 gestantes diagnosticadas com diabetes gestacional. 0 Estudo foi composto por dois grupos: das mães que foram diagnosticadas com DMG nesta gravidez pela primeira vez e das mães que, embora tenham tido DMG na gravidez anterior, conseguiram controlar a patologia, mas, na gravidez atual, desenvolveram a doença novamente.

Os dados da pesquisa foram coletados diariamente no período de agosto a outubro de 2011. Aaplicação do questionário se deu em um único momento. Os critérios de inclusão foram: as gestantes internadas com DMG no Setor de Obstetrícia do hospital, desde que a patologia tivesse desenvolvimento durante a gravidez; as gestantes que aceitaram participar da pesquisa e que estiveram em condições de responder ao questionário.

Os Critérios de Exclusão foram: gestantes não diabéticas internadas no hospital; as gestantes que foram atendidas, mas não estiveram internadas no referido Hospital; e aquelas que disseram ser portadora de DMG, entretanto, os prontuários não confirmaram.

Para atender aos objetivos da pesquisa foi utilizado como instrumento de coleta dos dados um questionário semiestruturado. No qual as perguntas fechadas foram utilizadas para atender ao primeiro objetivo da pesquisa por meio das variáveis: faixa etária, escolaridade, estado civil e renda familiar; História Gíneco-Obstétrica.

Para atender ao segundo objetivo foram utilizadas as perguntas abertas do questionário, relacionadas à percepção das gestantes sobre DMG.

As etapas da análise qualitativa foi compostas de quatro etapas:

1. Leitura geral da descrição que o sujeito relatou, nessa etapa as falas foram transcritas e realizou-se a leitura do todo buscando-se o sentido das falas das gestantes.

2. Compreensão das ideias ou sentido do todo, buscou-se ler novamente as falas para se identificar as unidades de sentido sobre o significado atribuídos pelas gestantes sobre DMG.

3. Depois de delineadas as unidades de sentido, buscou-se encontrar, mais diretamente, os aspectos contidos dentro dessas unidades relacionadas aos significados atribuídos pelas gestantes.

4. Por fim, chegou-se ao resultado, transformando todas as unidades de sentido em colocações consistentes sobre o significado atribuídos pelas gestantes sobre DMG. As unidades de sentido identificadas foram: Significados atribuídos pelas gestantes sobre Diabetes Mellitus Gestacional; Conhecimento da gestante sobre agravos a sua saúde e a do bebê; Mudanças desencadeadas pela DMG. Os resultados foram analisados a luz da literatura pertinente.

Para a análise quantitativa, utilizou-se a consolidação e análise dos dados quantitativos foram obtidas pelo programa estatístico SPSS (Statistical Analysis Sofware), que é um programa de análise de dados desenvolvido para a área da saúde e que possibilita o cruzamento de variáveis. No estudo qualitativo, a análise de conteúdo feita através da apreciação das categorias temáticas e organizada de acordo com as convergências.

$\mathrm{Na}$ análise, a população representada recebeu um símbolo, a letra $\mathrm{G}$, significando gestante, acompanhado com um número, que será a sequência de atendimentos a gestantes entrevistadas.

A pesquisa desenvolveu-se de acordo com a Resolução 466/2012 Comissão Nacional de Ética e Pesquisa (CONEP). E foi submetido ao comitê de ética em pesquisa da Universidade Estadual do Ceará, sob o parecer de nº 07199787.

\section{Resultados e discussões}

A população foi constituída por 17 mulheres, com faixa etária predominante entre 30 a 34 anos, 7 (41,2\%). Pode-se observar que todas as mulheres envolvidas no estudo se encontravam na faixa etária de risco. Esse é um dado bastante relevante, pois os profissionais de saúde devem estar alerta a todos os sinais e sintomas apresentados para evitar qualquer alteração orgânica nestas mulheres ${ }^{7}$.

Em relação ao estado civil, 15 (88,22\%), eram casadas ou possuíam alguma união estável, o que se mostrou um ponto positivo, pois contavam com o apoio familiar, condição imprescindível para uma recuperação adequada e satisfatória, além do amparo emocional.

Quanto à escolaridade observou-se que apenas uma das participantes era analfabeta. A baixa escolaridade, em muitos estudos, é considerada fator de risco, pois influencia na postura quanto a hábitos inadequados e prejudiciais à sua saúde.

A renda familiar das mulheres pesquisadas foi baixa, $8(47,0 \%)$ das participantes recebeu menos de um salário mínimo ( $R \$ 545,00$ ), o que se torna um dado alarmante e que pode contribuir negativamente para o tratamento da patologia, pois, nestas condições, fica difícil desenvolver uma vida saudável, devido à dificuldade em comprar alimentos benéficos à saúde e essenciais à manutenção das taxas glicêmicas normais, além das péssimas condições de moradia.

A tabela 1 mostra o perfil das participantes do estudo: 
Tabela 1 - Caracterização da população, segundo faixa etária, escolaridade, estado civil e renda familiar. Fortaleza, 2011.

\begin{tabular}{|c|c|c|}
\hline Faixa etária & $\mathbf{N}^{0}$ & $\%$ \\
\hline $20-24$ & - & - \\
\hline $25-29$ & 1 & 5,9 \\
\hline $30-34$ & 7 & 41,2 \\
\hline $35-39$ & 5 & 29,4 \\
\hline $40-45$ & 4 & 23,5 \\
\hline Total & 17 & 100,0 \\
\hline \multicolumn{3}{|l|}{ Escolaridade } \\
\hline Não alfabetizado & 1 & 5,9 \\
\hline Ensino fundamental & 7 & 41,2 \\
\hline Ensino médio & 9 & 52,9 \\
\hline Ensino superior & - & - \\
\hline Total & 17 & 100,0 \\
\hline \multicolumn{3}{|l|}{ Estado civil } \\
\hline Solteira & 2 & 11,8 \\
\hline Casado/ união estável & 15 & 88,2 \\
\hline Divorciada & - & - \\
\hline Viúva & - & - \\
\hline Total & 17 & 100,0 \\
\hline \multicolumn{3}{|l|}{ Renda familiar (SM) } \\
\hline Até 1 & 8 & 47,0 \\
\hline $1-2$ & 7 & 41,2 \\
\hline $2-3$ & 2 & 11,8 \\
\hline Acima de 3 & - & - \\
\hline Total & 17 & 100,0 \\
\hline
\end{tabular}

\section{História Gíneco-Obstétrica}

Quanto a recorrência de DMG, verifica-se na tabela 2 que $3(17,3 \%)$ das participantes relataram que também desenvolveram a doença (DMG) na gravidez anterior, e 14 (82,7\%) afirmaram que era a primeira vez que se deparavam com aquela problemática de saúde.

O período de acompanhamento das gestantes que receberam o diagnóstico de DMG pelos profissionais de saúde variou de 16 a 36 semanas. 0 diagnóstico de $6(35,4 \%)$ das participantes DMG ocorreu antes da $20^{2}$ semana de gestação. De acordo com a literatura, as mulheres que detectaram o diabetes gestacional antes da 24a semana, presumem-se que já tivessem a doença de forma latente, mas só tomou conhecimento na gravidez ${ }^{8}$.

Tabela 2 - Distribuição da população pesquisada segundo a recorrência de DMG. Fortaleza-CE, 2011.

\begin{tabular}{c|c|c}
\hline DMG & $\mathbf{N}^{0}$ & $\%$ \\
\hline Sim & 3 & 17,3 \\
\hline Não & 14 & 82,7 \\
\hline Total & 17 & 100,0 \\
\hline
\end{tabular}

O gráfico abaixo representa a frequência de Diabetes entre parentes das informantes. Das gestantes pesquisadas 11 $(64,7 \%)$ relataram que tinham parentes com diabetes; $6(35,29)$ dessas entrevistadas mencionou a mãe, fato relevante pois histórico familiar de diabetes em parentes de $1^{\circ}$ grau é fator de risco para o desencadeamento de $\mathrm{DMG}^{7}$.

Ainda em relação aos antecedentes familiares, $3(17,3 \%)$ informaram que não tem parentesco com pessoas diabéticas, isso é positivo, por ser um fator de risco bastante determinante para o desenvolvimento da patologia. Além disso, $3(17,3 \%)$ não sabiam informar seus antecedentes familiares, neste caso, é importante conversar com estas entrevistadas e despertar o interesse pelas patologias presentes em suas famílias para estarem cientes de seus acometimentos hereditários e, assim, ser possível tomar medidas preventivas como, por exemplo, mudança nos hábitos alimentares e pratica regular de exercícios. 
Outro dado importante também investigado, refere-se à macrossomia, característica comum em bebês de mulheres com DMG ${ }^{9}$. Essa ocorrência foi registrada em $6(35,3 \%)$ dos bebês das entrevistadas. A frequência de macrossômicos tem aumentado nas últimas décadas em diversos países sendo de 15 a $50 \%$ das gestações de pacientes portadoras com DMG ${ }^{10}$.

Uma pesquisa que objetivou identificar fatores maternos e perinatais, relacionados a fetos com peso igual ou maior do que 4.000 gramas no nascimento, detectou que complicações tendem a se associar ao excessivo tamanho fetal, e a macrossomia fetal permanece como um problema obstétrico de difícil solução, associado a importantes consequências maternas e perinatais, haja vista as significantes taxas de morbiletalidade observadas em países desenvolvidos e em desenvolvimento ${ }^{11}$.

Analisando os aspectos qualiquantitativos (tabela 3) relativos à assistência oferecida às gestantes, pode-se notar que $4(23,5 \%)$ tiveram uma quantidade adequada de consultas, conforme preconizada pelo Ministério da Saúde, que recomenda seis consultas para um bom acompanhamento pré-natal ${ }^{12}$.

Tabela 3 - Distribuição da população amostral segundo o número de consulta de pré-natal, Fortaleza- CE, 2011.

\begin{tabular}{c|c|c}
\hline $\mathbf{N}^{\circ}$ de consulta de pré-natal & $\mathbf{N}^{0}$ & $\%$ \\
\hline Nenhuma & 2 & 11,8 \\
\hline 2 a 3 consultas & 3 & 17,6 \\
\hline 4 a 5 & 8 & 47,1 \\
\hline 6 ou + & 4 & 23,5 \\
\hline Total & 17 & 100,0 \\
\hline
\end{tabular}

Quando questionadas sobre quais profissionais Ihes forneceram informação sobre DMG no pré-natal, verificou-se que $10(58,8 \%)$ das entrevistadas responderam que não tiveram nenhuma informação sobre a doença, apenas $3(17,6 \%$ das participantes declararam que receberam a informação com o enfermeiro; 3 (17,6\%), médico; e 1 (6\%) com o farmacêutico.

\section{Significados atribuídos pelas gestantes sobre Diabetes Mellitus Gestacional}

As falas das entrevistadas referentes ao conhecimento em relação à doença e seus cuidados foram selecionadas em unidades de sentido para proporcionar uma visão detalhada e clara do que foi relatado: negação absoluta do conhecimento falta de entendimento, obstáculo da escolaridade, negação e desejo, associação à gravidez.

Vale salientar que das 17 gestantes pesquisadas duas não tiveram nenhuma consulta de pré-natal. Além desse dado percebe-se que nos relatos as gestantes não fazem nenhuma referência ao pré-natal. 0 principal objetivo da atenção pré-natal é acolher a mulher desde o início da gravidez, assegurando, ao fim da gestação, o nascimento de uma criança saudável e a garantia do bem-estar materno e do recém-nascido ${ }^{12}$.

As falas das entrevistadas; G3, G8, G9, G10, G12, G13, G14, G15, G16 na categoria conhecimento em relação à doença: "não sei" e "não entendo", evidenciam a forma negativa de compreensão de sua patologia. 0 desconhecimento relatado pelas gestantes não significa, que não tenham recebido orientações por parte dos profissionais de saúde no período do pré-natal. Mas, representa a necessidade de se buscar estratégias educativas voltadas para as subjetividades inerentes a cada gestante para facilitar as ações preventivas de autocuidado e adesão ao pré-natal.

Uma das gestantes pesquisadas justificou não saber sobre a doença por ser iletrada: "Não entendo nada, sou analfabeta"(G1). Ser analfabeta não significa que uma pessoa não é dotada de um potencial de compreensão. É importante por meio de práticas educativas, resgatar os conhecimentos inerentes a gestante por meio de suas vivências cotidianas e relacioná-los a prevenção do DMG.

A educação em saúde é uma estratégia de promoção da saúde e prevenção de doenças e deve ser uma prática social do cuidado centrada nas questões do dia-a-dia dos indivíduos e grupos, na valorização das suas experiências, tendo como referência a realidade na qual estão inseridos ${ }^{13}$.

O cuidado em saúde deve estar pautado na autonomia dos usuários e profissionais da saúde. No processo de cuidado, deve-se abrir mão do controle, da propriedade das ações, pois os diferentes saberes devem ser levados em consideração. Para tanto é necessária a ruptura com o modo dominante e instituído de intervir sobre o território de saúde com dispositivos que interroguem sua lógica e exponha seu funcionamento, desnaturalizando e desestabilizando seus arranjos e significados para a construção de novas práticas em saúde e modos de produção de subjetividade ${ }^{14}$. 
Diante do desconhecimento expressado pelas gestantes portadoras de DMG sobre sua doença, uma gestante manifesta o desejo de entender o que estava acontecendo com sua saúde: "Não entendo, desejo entender" (G2). Percebe-se por meio deste relato a importância das atividades educativas no pré-natal.

A esse respeito autores referem que a educação em saúde no pré-natal é essencial para que a mulher desenvolvase a consciência da sua autonomia, "que compreenda a importância de realizar o pré-natal e identifique-o enquanto espaço de aprendizagem que oportuniza a mudança de práticas e condutas, resultando na adequação de novos hábitos e cuidados durante o ciclo gravídico e puerperal ${ }^{\prime \prime 5}$.

Uma gestante pesquisada referiu que nunca tinha ouvido falar da patologia antes e teve conhecimento da existência da doença somente no período da gravidez, mas de maneira muito falha, pois ela se mostrou impossibilitada de definir sua patologia: "Não tenho nem ideia, vim saber agora na gravidez, mas ainda não sei o que é" (G17).

Sabe-se que durante a consulta de pré-natal a gestante recebe muitas informações, mas isso não quer dizer que estão sendo aprendidas e que esteja ocorrendo mudança de comportamento. Para melhorar o nível de conhecimento das gestantes sobre a prevenção do diabetes gestacional é necessário o envolvimento de todos os envolvidos na construção da saúde, não somente a gestante, mas sua família, sua comunidade, os profissionais de diversas áreas de conhecimento e os gestores.

A transmissão do conhecimento em saúde deve estar articulado entre as diversas áreas de conhecimento, bem como os recursos existentes na comunidade devem ser mobilizados, como as escolas, igrejas, centros comunitários, associação de moradores, dentre recursos, para que seja possível, através da soma de esforços, maximizar o processo de educação em saúde, tornando o conhecimento mais acessível às populações, de forma que essas possam apropriarse de aspectos que contribuam para melhoria do seu estado de saúde ${ }^{16}$.

Neste relato, é possível perceber o conhecimento da transitoriedade da doença na gestação acompanhada da cura. Esse conceito é verdadeiro no qual o diabetes gestacional é ocasionado por intolerância aos carboidratos que ocorre na gravidez e desaparece depois do parto ${ }^{17}$, como constatamos na fala: "É a doença diabetes que é só no período da gestação e depois fica boa" (G4).

Em contradição, houve a seguinte afirmação: "A diabetes durante a gestação e depois pode continuar". (G11). Esse relato é mais voltado a prolongamento da doença depois do período gestacional, o que pode perfeitamente ocorrer em mulheres que já tenham tendências metabólicas e hereditárias para isso. Para aquelas pacientes de alto risco e que na consulta inicial de pré-natal já preenchem os critérios para diabetes fora da gestação serão classificadas não como DMG mas como DM ${ }^{17}$.

Uma definição afirmou que é uma patologia exclusiva da gravidez, sendo que a probabilidade de surgimento da doença aumentava na idade mais avançada, 40 anos: "O diabetes que desenvolve só na gestação e que tem probabilidade de desenvolver próximo aos 40 anos." (G6)

Percebe-se associação entre 0 aparecimento da enfermidade com a ingestão excessiva de açúcar e hiperglicemia como fator propiciador no desencadeamento da patologia "Quando come doce demais e a glicemia aumenta" (G5).

"É a doença que só acontece na gravidez, devido ao acúmulo de massa". (G7). Essa afirmação relata que a DMG é exclusiva da gravidez e origina-se pelo consumo exagerado de carboidratos complexos, no caso representado pela palavra "massa".

Um dado positivo é que algumas gestantes entrevistadas sabiam os fatores agravantes e alguns cuidados com a alimentação. Mas, para que uma gestante portadora de diabetes tenha uma gestação normal e fetos saudáveis, devem ser tomadas algumas precauções: planejar a gravidez; Controlar rigorosamente o nível de glicose no sangue; Detectar precocemente os fatores de risco, evitando suas complicações; hábitos de vida saudáveis e consultar regularmente 0 médico para adequar a dosagem de insulina ${ }^{11}$.

Considera-se diante dos achados dessa unidade de sentido que as percepções das gestantes, mapeiam os diversos significados inerentes a sua doença e que tais significados devem ser levados em consideração pelos profissionais de saúde durante o pré-natal, para que sejam aliados aos conhecimentos técnicos.

\section{Conhecimento da gestante sobre agravos a sua saúde e a do bebê}

Em relação ao conhecimento sobre agravos a sua saúde, foi bastante expressivo o número de mulheres que evidenciaram desconhecimento dos malefícios da patologia, não emitindo ao menos uma resposta ou um palpite sobre esse assunto como observamos na fala: "não sei". (G1), (G2), (G5), (G8), (G10), (G12), (G13), (G14), (G16). Isso é preocupante, pois pode pôr em risco a vida destas gestantes por não serem capazes de identificar o surgimento de alguma complicação.

Uma gestante refere que é o estado psicológico, "Creio que é psicológico." (G3), talvez por acreditar que acarrete alterações orgânicas e descompensação da glicemia. Além dos fatores relacionados à alteração da glicemia, as alterações psicológicas podem estar associadas às questões inerentes a gestante. 
Os fatores que desencadearam os sintomas desagradáveis do diabetes gestacional ao não planejamento da gravidez, a falta de apoio da família, sentimentos de medo, insegurança e impotência. $O$ apoio psicológico para a gestante e para a família é de fundamental importância para a aceitação da patologia ${ }^{18}$.

Houve também grande associação com a dieta inadequada, visão mais simplista e generalista e demonstrada nesta fala: "A alimentação" (G4), (G17), (G9), (G11). Isso mostra o quanto a alimentação desperta certo receio, por parte das gestantes como o principal fator agravante da DMG.

A ideia da alimentação saudável tem sido disseminada à população seja por meio dos meios de comunicação, seja por meio de profissionais especializados no assunto. Nesse sentido, em casos especiais de agravos a saúde é preciso que a alimentação faça parte da estratégia terapêutica de recuperação da saúde conduzida pelo profissional responsável pelo projeto terapêutico.

Os profissionais responsáveis pela educação devem ser treinados não somente em DM, mas principalmente em educação em saúde/nutrição².

Na DMG para que a alimentação seja adequada a terapia nutricional deve estar focada em escolhas alimentares que garantam um apropriado ganho de peso, controle glicêmico e ausência de corpos cetônicos ${ }^{19}$.

Uma fala mostrou bastante esclarecimento sobre os agravos da doença, listando uma série de complicações que pode sofrer o bebê e a gestante: "Parto prematuro, morte fetal, morte materna (hiperglicemia, hipoglicemia) o bebê corre 0 risco de nascer com DM e malformação" (G6)

Foi citado também como agravo a macrossomia, o que está certo, pois é o mais comum no DMG: "Aumenta o peso do bebe". (G7)

"Ter eclampsia. Causa morte da mãe e do filho". (G15) esta fala mostra agravos mais raros, mas que podem acontecer, acarretando efeitos mais graves ou até morte.

Com relação ao bebê, foi perguntado as gestantes se o DMG poderia causar algum problema de saúde a criança, das 17 gestantes 14 responderam que poderia causar problema ao bebê, mas a maioria não sabia dizer os principais danos. 0 que podemos observar na fala: "Não sei”. (G1), (G2), (G4), (G7), (G8), (G9), (G13), (G15), demonstrando uma grande falta de informação.

"Se é só na gravidez, então só a mãe é prejudicada". (G3), neste relato é perceptível o desconhecimento da gestante em relação aos danos do DMG ao filho, isso pode acarretar sequelas graves na criança e mostra também que ela considera a doença transitória e prejudicial somente à gestante.

"Se a diabetes não for tratada devidamente a criança pode nascer diabética e com má formação". (G11) Essa foi uma resposta muito interessante e consciente dos danos da doença que o bebê pode sofrer, e como a gestante é importante nisso, pois relata que a progenitora é responsável pelo controle da doença.

Foi mencionado também que o feto pode desenvolver má formação congênita, "Porque pode nascer com má formação". (G14). Isso pode acontecer em grávidas com Diabetes pré-existente, sendo mais raro em mulheres com DMG.

O receio que o filho sofra do mesmo mal pode ser notado no seguinte relato: "devido eu estar com a doença posso passar para ele". (G17).

Diante das percepções das gestantes sobre os agravos que o diabetes pode causar a sua saúde e a do feto, percebe-se que as causas pontuadas foram as mais significativas para as gestantes. Partido dos significados descritos pode-se direcionar as atividades de educação em saúde para fortalecimento do projeto terapêutico para o controle do diabetes gestacional.

\section{Mudancas desencadeadas pela DMG}

As principais mudanças na vida da portadora de DMG foram o estado psicológico e a alimentação, porém duas mulheres disseram que não mudaram nada.

Em relação ao estado psicológico, algumas disseram que ficaram mais preocupadas em desenvolver algum problema de saúde. Isso exige uma atuação dos profissionais de saúde mais voltada ao paciente e fazer compreender que é possível um bom prognóstico e um acompanhamento mais de perto e mais aprofundado destas gestantes. A equipe de saúde deve auxiliar a gestante a compreender o tratamento, e acreditar em um bom prognóstico materno-fetal ou até mesmo na cura. ${ }^{21}$

Houve pacientes que relataram que seu estado mental estava bom, mas estava apreensiva com a patologia; "na minha cabeça não mudou nada, só um pouco de preocupação" (G2). Também teve associação com preocupação, isso ocorreu, pois já havia casos na família de complicações avançadas de diabetes; "fiquei preocupada, porque é uma doença muito complicada, já teve muitos casos de amputação na família "Mudei de estilo de vida" (G4). "Fiquei preocupada, mudei a rotina de tudo (estilo de vida, dieta)" (G11), nesta fala podemos perceber que, apesar da preocupação, há iniciativa de mudar o estilo de vida, o que possibilitar um viver mais saudável.

Um estudo que avaliou a ansiedade e depressão em 20 gestantes que receberam o diagnóstico de DMG observou que $35 \%$ das gestantes diabéticas apresentaram depressão e 30\% apresentaram ansiedade. A concomitância de ansiedade e depressão foi observada em quatro das 20 pacientes $(20 \%)^{22}$. 
Mas houve três casos que disseram não ter tido uma mudança significativa na sua vida, como se nada tivesse acontecido.

"Não está sentindo mudanças no estilo de vida (G13)"

"Não mudou, porque eu não sentia nada e continuo não sentindo nada (G14)".

"Pra mim não mudou nada, porque o médico me falou que eu iria voltar ao normal com 45 dias após o parto" (G17).

$\mathrm{Na}$ última resposta a mudança não ocorreu, pois o médico disse que seu quadro voltaria ao normal.

Em relação à alimentação, praticamente a metade das participantes do estudo disse que mudou os hábitos alimentares, sendo que muitas não especificaram quais seriam estas alterações. O mais citado foi a redução da ingestão principalmente de doces e carboidratos: "Hábitos alimentares" (G3) (G6) (G7) (G8) (G10) (G11) (G16).

Diante do que foi exposto pelas gestantes os significados relacionados aos hábitos alimentares indicam que as gestantes compreendem, os malefícios do uso excessivo de açucares e carboidratos. Porém existe a possibilidade que certos tipos de amido, como os presentes na batata, no pão branco e em cereais matinais, flocos de milho, por exemplo, geram alterações glicêmicas maiores e mais rápidas do que até mesmo o açúcar ${ }^{20}$.

Daí a importância da participação mais ativa, efetiva e eficiente dos profissionais de saúde que vise uma detecção precoce e um tratamento adequado a estas mulheres.

\section{Considerações Finais}

A pesquisa possibilitou retratar um perfil socioeconômico, epidemiológico e clínico das grávidas com DMG atendidas na instituição em estudo, permitindo um maior conhecimento sobre as mulheres acometidas por esta enfermidade.

No estudo foi constatado que diante da identificação do perfil das gestantes estudadas encontrou-se vários fatores de risco para o desenvolvimento do diabetes gestacional tais como a idade em sua maioria com idade acima de 30 anos, a baixa renda familiar, a identificação do diabetes gestacional de modo tardio, bem como o início do pré-natal, sendo duas gestantes sem consulta de pré-natal, a ocorrência de macrossomia fetal.

Nos relatos detectou-se que alguns pontos devem ser priorizados pelos profissionais de saúde atuantes na atenção a gestante um dos fatores foi a falta de orientações adequadas durante o pré-natal, principalmente, diante do seu diagnóstico de diabetes gestacional. Muitas gestantes não sabiam a consequências do DMG e não mudaram seu estilo de vida para o controle glicêmico.

Nesses casos é necessária uma assistência pré-natal de maior qualidade, não se limitando apenas a exames complexos, mas que se refiram também aos aspectos psicológicos, antropológicos, sociais e culturais. Dessa forma a abordagem de uma gestante com DMG deve ser multidisciplinar e atuação dos profissionais de saúde é fundamental na prevenção e educação em saúde, visto que se trata de uma enfermidade complexa que demanda cuidados.

\section{Referências Bibliográficas}

1. Chaves EGS, Francisco PM, Nascentes GAN, Paschoini MC, Silva AP, Borges MF. Estudo retrospectivo das implicações maternas, fetais e perinatais em mulheres portadoras de diabetes, em 20 anos de acompanhamento no Hospital Escola da Universidade Federal do Triângulo Mineiro. Arquivo Brasileiro Endocrinologia \& Metabologia.2010; 54(7): 620-9.

2. Sociedade Brasileira de Diabetes. Cuidados de Enfermagem em Diabetes Mellitus. São Paulo: Editora do Departamento de Enfermagem da Sociedade Brasileira de Diabetes; 2014.

3. Ayach W, Calderon IMP, Rudge MVC, Costa RAA. Associação glicêmia de jejum e fatores de risco como teste para rastreamento do Diabetes Gestacional. Revista Brasileira de Saúde Materno Infantil. 2005; 5(3): 329-35.

4. Coutinho T, Coutinho CM, Duarte AMBR, Zimmermmann JB, Coutinho LM. Diabetes Gestacional: como tratar? Revista Femina. 2010; 38(10): 517-25.

5. Aquino MMA, Pereira BG, Amaral E, Parpinelli MA, Passini Jr R. Revendo diabetes e gravidez. Revista Ciência Médica. 2003;12(1):99-106.

6. Kenner C. Enfermagem neonatal. $2^{a}$ ed. Rio de Janeiro: Reichmann \& Affonso Editores; 2001.

7. Sociedade Brasileira de Diabetes. Atualização brasileira sobre diabetes. Rio de Janeiro: Diagraphic; 2006.

8. Mansur VAR, Laun IC, Farias MLF. Importância do rastreamento e diagnóstico do Diabetes Gestacional. Revista Femina. 2001; 29(3): 143-7. 
9. Corrêa FHS, Gomes MB. Acompanhamento ambulatorial de gestantes com DM no hospital universitário Pedro Ernesto -UERJ. Arquivo Brasileiro Endocrinologia \& Metabologia. 2004; 48(4):499-504.

10. Amorim MMR, Leite DFB, Gadelha TGN, Muniz AGV, Melo ASO, Rocha AM. Fatores de risco para macrossomia em recém-nascidos de uma maternidade-escola no nordeste do Brasil. Rev. Bras. Ginecol. 2009. 31(5): 241-8.

11. Brasil. Ministério da Saúde. Pré-natal e Puerpério: atenção qualificada e humanizada: manual técnico. Brasilia: Ministério da Saúde, 2005.

12. Câmara, AMCS. et al. Percepção do Processo Saúde-doença: Significados e Valores da Educação em Saúde. Revista Brasileira de Educação Médica,v. 36,1 Supl. 1,p. 40 - 50, 2012.

13. Madi JM, Rombaldi RL, Oliveira Filho PF, Araujo BF, Zatti H, Madi SRC.Fatores maternos e perinatais relacionados à macrossomia fetal. Revista Brasileira de Ginecologia e Obstetrícia.2006; 28(4): 232-7.

14. Fortuna, CM. et al. O enfermeiro e as práticas de cuidados coletivos na estratégia saúde da família. Rev. Latino-Am. Enfermagem 19(3):[08 telas] maio-jun 2011.

15. Carneiro, MS. et al.Representações sociais sobre pré-natal entre mulheres-mães: implicações para o agir cuidativo educativo em enfermagem. Enfermagem Obstétrica, Rio de Janeiro, 2014 jan/abr; 1(1):12-8.

16. Borges, TT. et al. Prevalência de autorrelato da morbidade e conhecimento sobre diabetes: estudo populacional de uma cidade no sul do Brasil. Rev. bras. cineantropom. desempenho hum., v.14, n.5, p. 562-570, 2012.

17. SBD. Sociedade Brasileira de Diabetes. Diretrizes da Sociedade Brasileira de Diabetes: 2013-2014/Sociedade Brasileira de Diabetes ; [organização José Egidio Paulo de Oliveira, Sérgio Vencio]. - São Paulo: AC Farmacêutica, 2014. 18. Mundim, RAS. Sentimentos vivenciados pelas gestantes portadoras de diabestes mellitus. Revista de Biotecnologia \& Ciência Vol. 2, No. 1, Ano 2011 p. 62-82.

19. Padilha, PC. et al. Terapia nutricional no diabetes gestacional. Rev. Nutr. 2010, vol.23, n.1, pp. 95-105.

20. Barreto, SM. et al. Análise da Estratégia Global para Alimentação, Atividade Física e Saúde, da Organização Mundial da Saúde. Epidemiologia e Serviços de Saúde, V.14, N. 1, jan/mar, 2005.

21. Silva L, Santos RC, Parada CMGL. Compreendendo o significado da gestação para grávidas diabéticas. Revista Latino-Americana de Enfermagem. 2004; 12(6):899-904.

22. Pessoa SMF, Pagliuca LMF, Damasceno MMC. Teoria do cuidado humano: análise crítica e possibilidades de aplicação a mulheres com Diabetes Gestacional. Revista de Enfermagem da UERJ. 2006;14(3):463-9.

\section{Rosiana Carvalho Costa}

Endereço para correspondência - Rua Alexandre Barauna, № 1330, Bairro: Rodolfo Teófilo, CEP: 60430-160, Cidade: Fortaleza, CE, Brasil.

E-mail: Rosi.Carvalho@gmail.com

Lattes: http://lattes.cnpq.br/2798772401808289

Márcia Oliveira Coelho Campos - lidiavaladas@hotmail.com

Lidia Audrey Rocha Valadas Marques - lidiavaladas@gmail.com

Edilson Martins Rodrigues Neto - edilsonmrneto@gmail.com

Maria Celsa Franco - lidiarvm@gmail.com

Érika Sabóia Guerra Diógenes - erikadiogenes@hotmail.com

Enviado em 12 de abril de 2014. Aceito em 11 de dezembro de 2014. 
